\title{
ON THERMOSOLUTAL CONVECTION IN PRESENCE OF COMPRESSIBLE FLUID WITH FINE DUST
}

\author{
M. SINGH* \\ Department of Mathematics \\ Govt Post Graduate College, Seema-Rohru \\ Himachal Pradesh University Summer-Hill \\ Shimla-171207, INDIA \\ E-mail: mahinder_singh91@Rediffmail.in \\ R.K. GUPTA \\ Department of Mathematics \\ Lovely School of Engineering and Technology \\ Lovely Professional University \\ Phagwara, INDIA \\ E-mail: rkgupta2003@Indiatimes.com
}

\begin{abstract}
A layer of a Rivlin-Ericksen elastico-viscous fluid heated and soluted from below in the presence of compressibility and suspended particles (fine dust) effect is considered. For stationary convection, the RivlinEricksen, elastico-viscous fluid behaves like a Newtonian fluid. The oscillatory modes are introduced due to the presence of a stable solute gradient, suspended particles destabilize the system whereas the stable solute gradient has a stabilizing effect on the system and the effect of compressibility is to postpone the onset of thermosolutal convection. The stable solute gradient and compressibility postpone the onset of convection, whereas the suspended particles hasten the onset of convection. The stable solute gradient introduces oscillatory modes in the systems which were non-existent in its absence.
\end{abstract}

Key word: Rivlin-Ericksen elastico-viscous fluids, compressible and fine dust.

\section{Introduction}

A layer of a Newtonian fluid heated from below, under varying assumptions of hydrodynamics, has been treated in detail by Chandrasekhar (1981). Chandra (1938) performed careful experiments in an air layer and found a contradiction between the theory and the experiment. He found that the instability depended on the depth of the layer. A Benard- type cellular convection with the fluid descending at the cell centre was observed when predicted gradients were imposed, if the layer depth was more than $10 \mathrm{~mm}$. But if the layer of depth was less than $7 \mathrm{~mm}$, convection occurred at much lower gradients than predicted and appeared as irregular strips of elongated cells with the fluid rising at the centre. Chandra called this motion columnar instability. The effect of particle mass and heat capacity on the onset of Ben'ard convection was considered by Scanlon and Segel (1973). They found that the critical Rayleigh number was reduced solely because the heat capacity of the clean gas was supplemented by that of the particles. The effect of suspended particles was found to destabilize the layer. Palniswamy and Purushottam (1981) considered the stability of shear flow of stratified fluids with fine dust and found that the effect of fine dust increases the region of instability. A study of double-diffusive convection with fine dust was made by Sharma and Rani (1989).

\footnotetext{
* To whom correspondence should be addressed
} 
The stability of the plane interface separating two Rivlin-Ericksen elastico-viscous superposed fluids was studied by Kumar at el. (2007). The density, viscosity and viscoelasticity are assumed to be, for stable stratifications, the system is found to be stable or unstable under certain conditions. Kumar at el. (2006) studied that effect of a magnetic field on thermal instability of a rotating Rivlin-Ericksen visco-elastic fluid, in which the magnetic field has a stabilizing as well as destabilizing effect on the system. Also, RayleighTaylor instability of a Rivlin-Ericksen elastico-viscous fluid through a porous medium was considered by Sharma at el. (2001). They studied the stability aspects of the system. The effects of a uniform horizontal magnetic field and uniform rotation on the problem was also considered separately. Kumar (2000) also studied the stability of superposed viscous-viscoelastic Rivlin-Ericksen fluids in the presence of suspended particles through a porous medium. In another study Kumar and Singh (2010) studied the stability of superposed viscous-viscoelastic fluids through a porous medium, and considered the effect of a uniform horizontal magnetic field and uniform rotation. Singh and Gupta (2011) studied the thermal instability of a Rivlin-Ericksen elastico viscous fluid permeated with suspended particles in a porous medium.

Sharma et al. (2006) studied the stability of a stratified elastico-viscous Walter's (B') fluid in the presence of a horizontal magnetic field and rotation in a porous medium.

\section{Formulation of the problem and perturbation equations}

Consider an infinite horizontal Rivilin-elastico-viscous fluid layer, heated with suspended particles, of depth $d$. This layer is heated and soluted from below so that the temperature, the solute concentration and density at the bottom surface $z=0$ are $T_{0}, C_{0}$ and $\rho_{0}$, respectively. A steady adverse temperature gradient $\beta\left(=\left|\frac{d T}{d z}\right|\right)$ and solute gradient $\beta^{\prime}\left(=\left|\frac{d C}{d z}\right|\right)$ are maintained. The system is acted on by the gravity force $\boldsymbol{g}(0,0,-g)$. The effect of fluid compressibility, even small in magnitude is also considered.

Let $\rho, \mu, \mu^{\prime}$ and $\boldsymbol{u}(u, v, w)$ denote the density, viscosity, viscoelasticity and velocity of the pure fluid, $\boldsymbol{v}(\boldsymbol{x}, t)$ and $N(\boldsymbol{x}, t)$ denote the velocity and number density of the suspended particles, $K=6 \pi \mu \eta$, is the Stokes drag coefficient $\eta$ being particle radius. Then the equations of motion and continuity governing the flow are

$$
\rho\left[\frac{\partial \boldsymbol{u}}{\partial t}+(\boldsymbol{u} \cdot \nabla) \boldsymbol{u}\right]=-\nabla p+\rho \boldsymbol{g}+K N(\boldsymbol{v}-\boldsymbol{u})+\left(\mu+\mu^{\prime} \frac{\partial}{\partial t}\right) \nabla^{2} \boldsymbol{u}
$$

and

$$
\nabla \cdot \boldsymbol{u}=0
$$

Assuming a uniform particle size, spherical shape and small relative velocities between the fluid and particles, the presence of particles adds an extra force term in the equation of motion (1), proportional to the velocity difference between the particles and the fluid. The force exerted by the fluid on the particles is equal and opposite to that exerted by the particles on the fluid. The distance between the particles is assumed quite large compared with their diameters, so that interparticle reactions are ignored. The buoyancy forces on the particles are neglected. If $m N$ is the mass of the particles per unit volume, then the equations of motion and continuity for the particles, under the above assumptions, are

$$
m N\left[\frac{\partial \boldsymbol{v}}{\partial t}+(\boldsymbol{v} \cdot \nabla) \boldsymbol{v}\right]=K N(\boldsymbol{u}-\boldsymbol{v}),
$$

and 


$$
\frac{\partial N}{\partial t}+\nabla \cdot(N v)=0
$$

Let $C_{v}, C_{p t}, C_{p}, T$ and $q$ denote, the fluid at constant volume, heat capacity of particles, and heat capacity of the fluid at constant pressure, temperature and effective thermal conductivity of the pure fluid. Hence the volume fractions of the particles are assumed small, the effective properties of the suspension are taken to be those of the clean fluid. If we assume that the particles and fluid are in the thermal equilibrium, the equation of heat conduction gives

$$
\rho C_{v}\left[\frac{\partial}{\partial t}+\boldsymbol{u} \cdot \nabla\right] T+m N C_{p t}\left(\frac{\partial}{\partial t}+\boldsymbol{v} \cdot \nabla\right) T=q \nabla^{2} T .
$$

If $C$ denotes the solute concentration, the equation of solute conduction gives

$$
\rho C_{v}^{\prime}\left[\frac{\partial}{\partial t}+\boldsymbol{u} \cdot \nabla\right] C+m N C_{p t}^{\prime}\left[\frac{\partial}{\partial t}+\boldsymbol{v} \cdot \nabla\right] C=q^{\prime} \nabla^{2} C
$$

where $C_{v}^{\prime}, C_{p t}^{\prime}$ and $q^{\prime}$ denote analogous solute quantities.

Spiegel and Veronis (1960) defined $f$ as any one of the state variables (pressure $p$, density $\rho$ or temperature $T$ ) and expressed it in the form

$$
f(x, y, z, t)=f_{m}+f_{0}(z)+f^{\prime}(x, y, z, t)
$$

where $f_{m}$ is the constant space average of $f, f_{0}$ is the variation in the absence of motion and $f^{\prime}$ is the fluctuation resulting from motion.

The initial state is therefore a state in which the density, pressure, temperature, solute concentration and velocity at any point in the fluid are given by

$$
\rho=\rho(z), \quad p=p(z), \quad T=T(z), \quad C=C(z), \quad \boldsymbol{v}=0, \quad \boldsymbol{u}=0, \quad N=N_{0},
$$

constant respectively, where

$$
\begin{aligned}
& T(z)=T_{0}-\beta z, \quad C(z)=C_{0}-\beta^{\prime} z, \\
& P(z)=p_{m}-g \int_{0}^{z}\left(\rho_{m}-\rho_{0}\right) d z, \\
& \rho(z)=\rho_{m}\left[1-\alpha_{m}\left(T-T_{m}\right)+\alpha_{m}^{\prime}\left(C-C_{m}\right)+K_{m}\left(p-p_{m}\right)\right], \\
& \alpha_{m}=-\left(\frac{1}{\rho} \frac{\partial \rho}{\partial T}\right)_{m}(=\alpha \text { say }), \quad \alpha_{m}^{\prime}=-\left(\frac{1}{\rho} \frac{\partial \rho}{\partial C}\right)_{m}\left(=\alpha^{\prime} \text { say }\right) \\
& K_{m}=-\left(\frac{1}{\rho} \frac{\partial \rho}{\partial p}\right)_{m} .
\end{aligned}
$$


Consider a small perturbation on the steady state solution and let $\delta p, \delta \rho, \theta, \gamma, \boldsymbol{u}(u, v, w), \boldsymbol{v}(1 . r, s)$ and $N$ denote respectively the perturbations in the pressure $p$, density $\rho$, temperature $T$, solute concentration $C$, fluid velocity $\boldsymbol{u}(0,0,0)$, particle velocity $\boldsymbol{v}(0,0,0)$ and number density $N_{0}$. The change in the density $\delta \rho$ , caused mainly by the perturbations $\theta$ and $\gamma$ in temperature and solute concentration, is given by

$$
\delta \rho=-\rho_{m}\left(\alpha \theta-\alpha^{\prime} \gamma\right)
$$

Then the linearized perturbation equations relevant to the problem (Spiegel and Veronis, 1960; Scanlon and Segel, 1973; Rivlin and Ericksen, 2001) become

$$
\begin{aligned}
& \frac{\partial \boldsymbol{u}}{\partial t}=-\frac{1}{\rho_{m}} \nabla \delta p+g\left(\alpha \theta-\alpha^{\prime} \gamma\right) \lambda+\frac{K N}{\rho_{m}}(\boldsymbol{v}-\boldsymbol{u})+\left(v+v^{\prime} \frac{\partial}{\partial t}\right) \nabla^{2} \boldsymbol{u} \\
& \nabla \cdot \boldsymbol{u}=0 \\
& \left(\frac{m}{K} \frac{\partial}{\partial t}+1\right) \boldsymbol{v}=\boldsymbol{u} \\
& \frac{\partial N}{\partial t}+\nabla \cdot\left(N_{0} v\right)=0 \\
& (1+h) \frac{\partial \theta}{\partial t}=\beta\left(\frac{G-1}{G}\right)(w+h s)+\kappa \nabla^{2} \theta \\
& \left(1+h^{\prime}\right) \frac{\partial \gamma}{\partial t}=\beta^{\prime}\left(w+h^{\prime} s\right)+\kappa^{\prime} \nabla^{2} \gamma
\end{aligned}
$$

where $\mu, \mu^{\prime}, v=\frac{\mu}{\rho_{m}}, v^{\prime}=\frac{\mu^{\prime}}{\rho_{m}}, \kappa\left(=\frac{q}{\rho_{m} C_{v}}\right)$ and $\kappa^{\prime}\left(=\frac{q^{\prime}}{\rho_{m} C_{v}^{\prime}}\right)$ stand for viscosity, viscoelasticity, kinematic viscosity, kinematic viscoelasticity, thermal diffusivity and analogous solute diffusivity, respectively.

Also,

$$
h=f \frac{C_{p t}}{C_{v}}, h^{\prime}=f \frac{C_{p t}^{\prime}}{C_{v}^{\prime}}, f=\frac{m N_{0}}{\rho_{m}} \text { and } G=\frac{C_{p} \beta}{g} .
$$

Initially, $\boldsymbol{u}=(0,0,0), \boldsymbol{v}=(0,0,0), T=T(z), N=N_{0}$, so Eq.(2.5) yields $0=0$, identically. After perturbation (2.5) becomes

$$
\left(\rho_{m}+\delta \rho\right) C_{v}\left(\frac{\partial}{\partial t}+\boldsymbol{u} \cdot \nabla\right)(T+\theta)+\left(m N_{0}+m N\right) C_{p t}\left(\frac{\partial}{\partial t}+\boldsymbol{v} \cdot \nabla\right)(T+\theta)=q \nabla^{2}(T+\theta) .
$$

Following Speigal and Veronis (1960) wherein the flow equations are found to be the same as those for incompressible fluids except that the static temperature gradient is replaced by its excess over the adiabatic and $C_{v}$ is replaced by $C_{p}$, i.e., $\beta$ is replaced by $\left(\beta-\frac{g}{C_{p}}\right)$, and linearizing Eq.(2.5) gives 


$$
\frac{\partial \theta}{\partial t}+\frac{m N_{0}}{\rho_{m}} \frac{C_{p t}}{C_{v}} \frac{\partial \theta}{\partial t}=\left(\beta-\frac{g}{C_{p}}\right)(w+h s)+\frac{q}{\rho_{m} C_{v}} \nabla^{2} \theta
$$

i.e., Eq.(2.13). However, $\beta^{\prime}$ remains unaltered and following the same procedure as above, Eq.(2.6) yields Eq.(2.14).

\section{The dispersion relation}

Analyzing the disturbances into normal modes, we assume that the perturbation quantities are of the form

$$
[w, \theta, \gamma]=[W(z), \Theta(z), \Gamma(z)] \exp \left[i k_{x} x+i k_{y} y+n t\right],
$$

where $k_{x}$ and $k_{y}$ are wave numbers along $x$-and $y$-directions respectively, $k\left(=\sqrt{k_{x}^{2}+k_{y}^{2}}\right)$ is the resultant wave number and $n$ is the growth rate, which is, in general a complex constant. Using (2.15), Eqs (2.9)(2.14) in a non-dimensional form become

$$
\begin{aligned}
& {\left[\sigma\left(1+\frac{M}{1+\tau_{1}}\right)-(1+F \sigma)\left(D^{2}-a^{2}\right)\right]\left(D^{2}-a^{2}\right) W+\frac{g a^{2} d^{2}}{v}\left(\alpha \theta-\alpha^{\prime} \Gamma\right)=0,} \\
& \left(D^{2}-a^{2}-H p_{1} \sigma\right) \Theta=-\beta\left(\frac{G-1}{G}\right) \frac{d^{2}}{\kappa} \frac{\left(H+\tau_{l} \sigma\right)}{\left(1+\tau_{l} \sigma\right)} W, \\
& \left(D^{2}-a^{2}-H^{\prime} q \sigma\right) \Gamma=-\beta^{\prime} \frac{d^{2}}{\kappa^{\prime}} \frac{\left(H^{\prime}+\tau_{l} \sigma\right)}{\left(1+\tau_{l} \sigma\right)} W
\end{aligned}
$$

where we have put $a=k d$,

$$
\begin{aligned}
& \sigma=\frac{n d^{2}}{\nu}, \quad \tau=\frac{m}{\kappa}, \quad \tau_{l}=\frac{\tau \nu}{d^{2}}, \quad M=\frac{m N}{\rho_{m}}, \quad p_{1}=\frac{\nu}{\kappa}, \\
& q=\frac{\nu}{\kappa^{\prime}}, \quad H=1+h, \quad H^{\prime}=1+h^{\prime}, \quad F=\frac{\nu^{\prime}}{d^{2}},
\end{aligned}
$$

and

$$
D=\frac{d}{d z}
$$

Eliminating $\Theta$ and $\Gamma$ between Eqs (3.2) and (3.4), we obtain

$$
\begin{aligned}
& {\left[\sigma\left(1+\frac{M}{1+\tau_{l} \sigma}\right)-(1+F \sigma)\left(D^{2}-a^{2}\right)\right]\left(D^{2}-a^{2}-H p_{1} \sigma\right)\left(D^{2}-a^{2}-H^{\prime} q \sigma\right)\left(D^{2}-a^{2}\right) W+} \\
& -R\left(\frac{G-1}{G}\right) a^{2}\left(\frac{H+\tau_{l} \sigma}{1+\tau_{l} \sigma}\right)\left(D^{2}-a^{2}-H^{\prime} q \sigma\right) W+S a^{2}\left(\frac{H^{\prime}+\tau_{l} \sigma}{1+\tau_{l} \sigma}\right)\left(D^{2}-a^{2}-H p_{l} \sigma\right) W=0
\end{aligned}
$$


where $R=\frac{g \alpha \beta d^{4}}{v \kappa}$ is the thermal Rayleigh number,

$$
\begin{aligned}
& S=\frac{g \alpha^{\prime} \beta^{\prime} d^{4}}{\nu \kappa^{\prime}} \text { is the analogous solute Rayleigh number, } \\
& p_{1}=\frac{\nu}{\kappa} \text { is the thermal Prandtl number }
\end{aligned}
$$

and

$$
q=\frac{\nu}{\kappa^{\prime}} \text { is the analogous Schmidt number. }
$$

We consider the case where both the boundaries are free and perfect conductors of heat, solute, while the adjoining medium is assumed to be electrically non-conducting. The appropriate boundary conditions for the case are

$$
W=D^{2} W=\Theta=\Gamma=D Z=0 \quad \text { at } \quad z=0 \quad \text { and } \quad 1 .
$$

The case of two free boundaries is the most appropriate. Using Eq.(3.6), we can show that all the even order derivatives of $W$ must vanish for $z=0$ and 1 and hence the proper solution of $W$ characterizing the lowest mode is

$$
W=W_{0} \sin \pi z
$$

where $W_{0}$ is a constant.

Substituting (3.7) in Eq.(3.5), we obtain the dispersion relation

$$
\begin{aligned}
& R_{l} x=\left(\frac{G}{G-1}\right)\left[\left\{i \sigma_{1}\left(1+\frac{M}{1+i \tau_{l} \sigma \pi^{2}}\right)+\left(1+i F \sigma \pi^{2}\right)(1+x)\right\}\right. \\
& \left.\left\{\frac{\left(1+i \tau_{1} \sigma \pi^{2}\right)(1+x)\left(1+x+i H p_{1} \sigma\right)}{H+i \tau_{l} \sigma \pi^{2}}\right\}+\frac{S_{l} x\left(H^{\prime}+i \tau_{1} \sigma \pi^{2}\right)\left(1+x+i H p_{l} \sigma\right)}{\left(H+i \tau_{l} \sigma \pi^{2}\right)\left(1+x+i H^{\prime} q \sigma\right)}\right]
\end{aligned}
$$

where

$$
R_{l}=\frac{R}{\pi^{4}}, \quad x=\frac{a^{2}}{\pi^{2}}, \quad i \sigma_{1}=\frac{\sigma}{\pi^{2}}, \quad S_{1}=\frac{S}{\pi^{4}} .
$$

Equation (3.8) is the required dispersion relation studying the effects of suspended particles and compressibility on the thermosolutal convection in the Rivlin-Ericksen elastico- viscous fluid. 


\section{The stability of the system and oscillatory modes}

Here, we examine whether instability can occur as oscillatory modes, if any, on the Rivlin-Ericksen elastico-viscous fluid in the presence of compressibility and suspended particles effects.

Multiplying Eq.(3.2) by $W^{*}$, the complex conjugate of $W$, integrating over the range of $z$ and making use of Eqs (3.3) and (3.4) with the help of boundary conditions (3.6), we obtain

$$
\begin{aligned}
& \sigma=\left(1+\frac{M}{1+\tau_{l} \sigma}\right) I_{1}+(1+F \sigma) I_{2}-\frac{g \alpha a^{2} \kappa}{v \beta}\left(\frac{G}{G-1}\right)\left(\frac{1+\tau_{l} \sigma^{*}}{H+\tau_{l} \sigma^{*}}\right)\left(I_{3}+H p_{1} \sigma^{*} I_{4}\right)+ \\
& +\frac{g \alpha^{\prime} a^{2} \kappa^{\prime}}{v \beta^{\prime}}\left(\frac{1+\tau_{l} \sigma^{*}}{H^{\prime}+\tau_{l} \sigma^{*}}\right)\left(I_{5}+H^{\prime} q \sigma^{*} I_{6}\right)=0
\end{aligned}
$$

where

$$
\begin{array}{ll}
I_{1}=\int_{0}^{1}\left(|D W|^{2}+a^{2}|w|^{2}\right), & I_{2}=\int_{0}^{1}\left(\left|D^{2} W\right|^{2}+2 a^{2}|D w|^{2}+a^{4}|W|^{2}\right), \\
I_{3}=\int_{0}^{1}\left(|D \Theta|^{2}+a^{2}|\Theta|^{2}\right) d z, & I_{4}=\int_{0}^{1}|\Theta|^{2} d z, \\
I_{5}=\int_{0}^{1}\left(|D \Gamma|^{2}+a^{2}|\Gamma|^{2}\right) d z, & I_{6}=\int_{0}^{1}|\Gamma|^{2} d z .
\end{array}
$$

The integrals $I_{1}-I_{6}$ are all positive definite. Putting $\sigma=i \sigma_{i}$, where $\sigma_{i}$ is real and equating the imaginary parts, we obtain

$$
\begin{aligned}
& \sigma_{i}=\left[\left(1+\frac{M}{1+\tau_{l} \sigma_{i}}\right) I_{1}+F I_{2}+\frac{g \alpha a^{2} \kappa}{v \beta}\left(\frac{G}{G-1}\right)\left(\frac{\tau_{l}(H-l)}{H^{2}+\tau_{l}^{2} \sigma_{i}^{2}} I_{3}+\frac{H+\tau_{l}^{2} \sigma_{i}^{2}}{H^{2}+\tau_{l}^{2} \sigma_{i}^{2}} H p_{l} \sigma^{*} I_{4}\right)+\right. \\
& \left.-\frac{g \alpha^{\prime} a^{2} \kappa^{\prime}}{v \beta^{\prime}}\left(\frac{\tau_{l}\left(H^{\prime}-1\right)}{H^{\prime 2}+\tau_{l}^{2} \sigma_{i}^{2}} I_{5}+\frac{H^{\prime}+\tau_{l}^{2} \sigma_{i}^{2}}{H^{\prime 2}+\tau_{l}^{2} \sigma_{i}^{2}} H^{\prime} q \sigma^{*} I_{6}\right)\right]=0 .
\end{aligned}
$$

Equation (4.2) implies that $\sigma_{i}=0$ or $\sigma_{i} \neq 0$, which means that the modes may be non-oscillatory or oscillatory. The oscillatory modes are introduced due to the presence of the stable solute gradient, and were non-existent in its absence.

\section{The stationary convection}

When instability sets in as stationary convection, the marginal state will be characterized by $\sigma=0$. Putting $\sigma=0$ in Eq.(3.8), the dispersion relation reduces to 


$$
R_{1}=\left(\frac{G}{G-1}\right)\left[\frac{(1+x)^{3}}{x H}+S_{1} \frac{H^{\prime}}{H}\right]
$$

and the Rivlin-Ericksen elastico- viscous fluid behaves like an ordinary Newtonian fluid.

To study the effect of suspended particles and the stable solute gradient, we examine the behaviour of $\frac{d R_{1}}{d H}$ and $\frac{d R_{1}}{d S_{1}}$ analytically.

Equation (5.1) yields

$$
\frac{d R_{1}}{d H}=-\left(\frac{G}{G-1}\right)\left[\frac{(1+x)^{3}}{x}+S_{1} H^{\prime}\right] \frac{1}{H^{2}},
$$

which is negative, implying thereby that the Rayleigh number decreases with an increase in the suspended particles number density. Therefore, suspended particles destabilize the system.

$$
\frac{d R_{1}}{d S_{1}}=\left(\frac{G}{G-1}\right) \frac{H^{\prime}}{H}
$$

is positive, thereby the Rayleigh number increases with an increase in the solute parameter. The stable solute gradient, therefore, has a stabilizing effect on the system.

Graphically, it is shown in Figs 1-3. In Figs 1 and 3 as values of $S_{1}$ and $H^{\prime}$ increase the values of $R_{l}$ increase to show that the stabilizing effects of the system. On the other hand as the values of $H$ increase the values of $R_{I}$ decrease to show the destabilizing effects of the system.

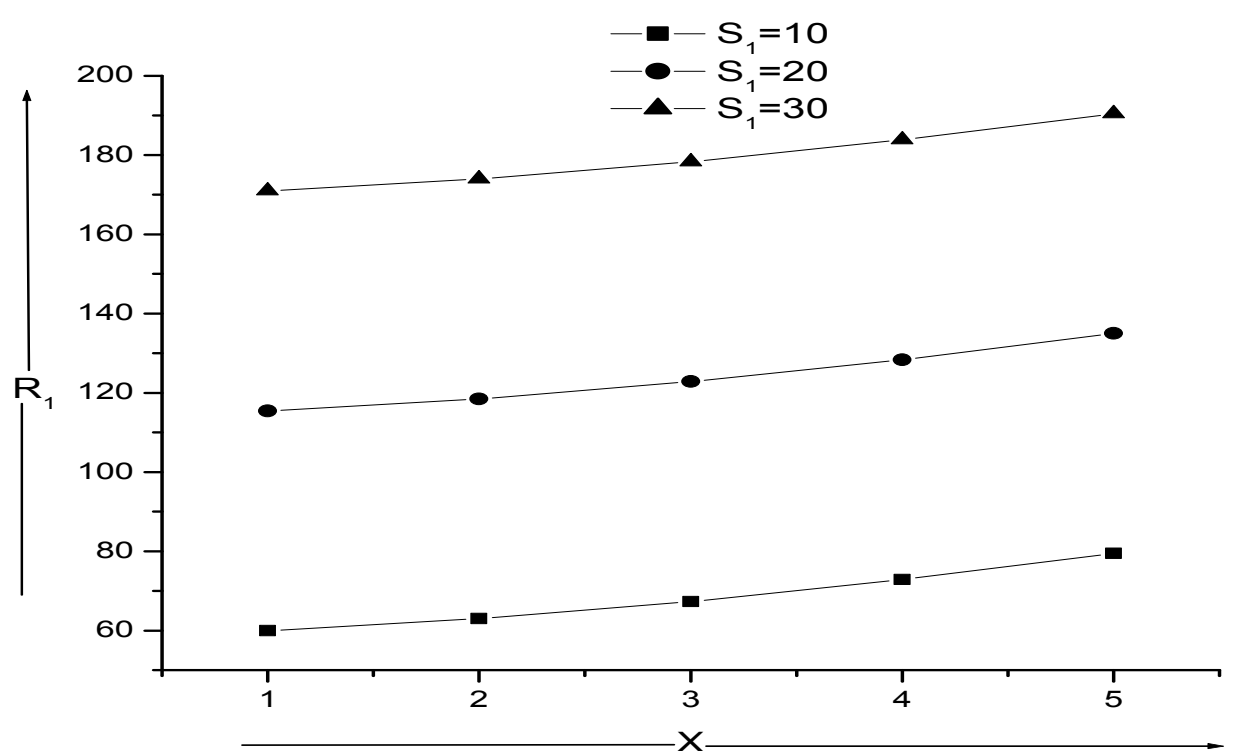

Fig.1. The variation of the Rayleigh number $\left(\mathrm{R}_{1}\right)$, with the wave number $x(=1,2,3,4,5)$, for $G=9.8$, $H=2, H^{\prime}=10$ and $S_{1}(=10,20,30)$. 


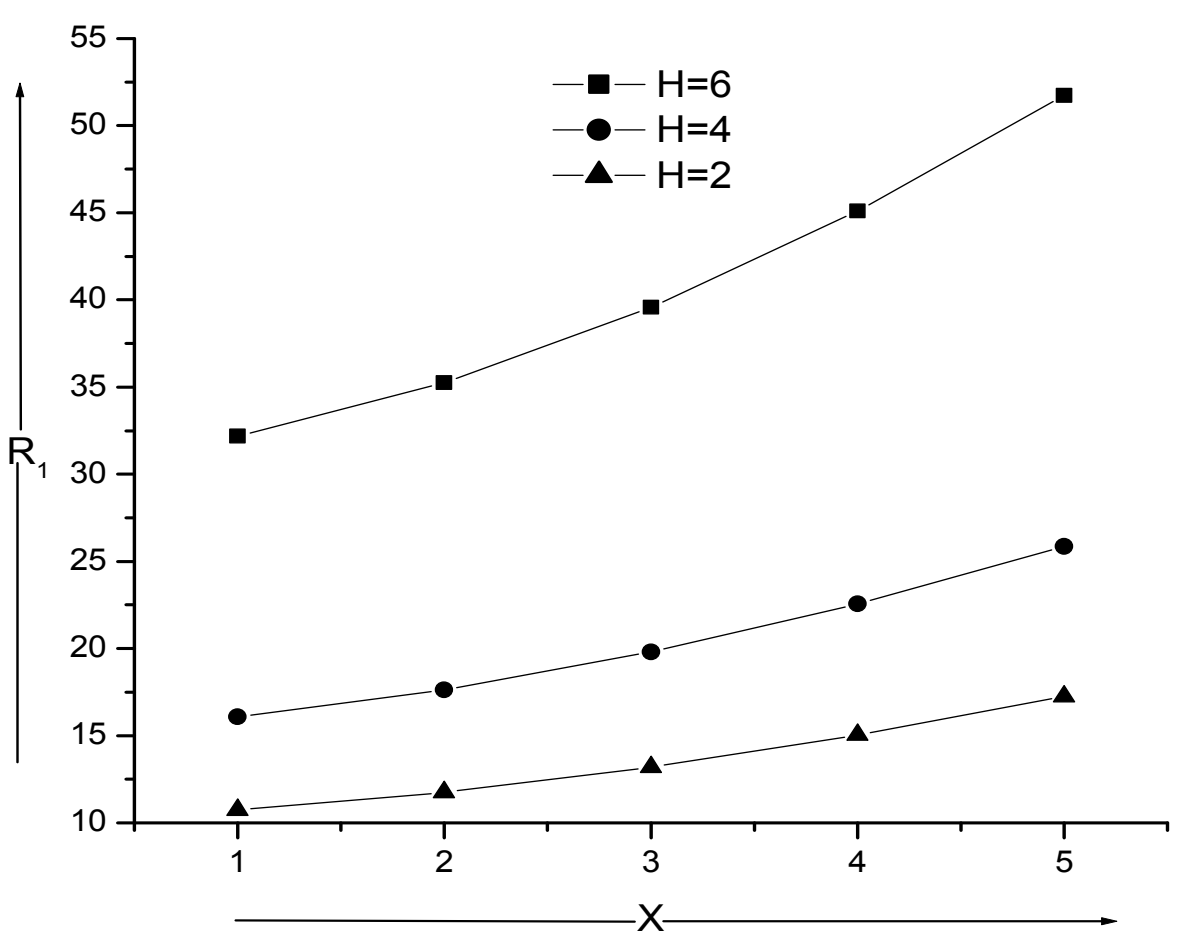

Fig.2. The variation of the Rayleigh number $\left(\mathrm{R}_{1}\right)$, with the wave number $x(=1,2,3,4,5)$, for $G=9.8$, $S_{1}=10, H^{\prime}=5$ and $H(=2,4,6)$.

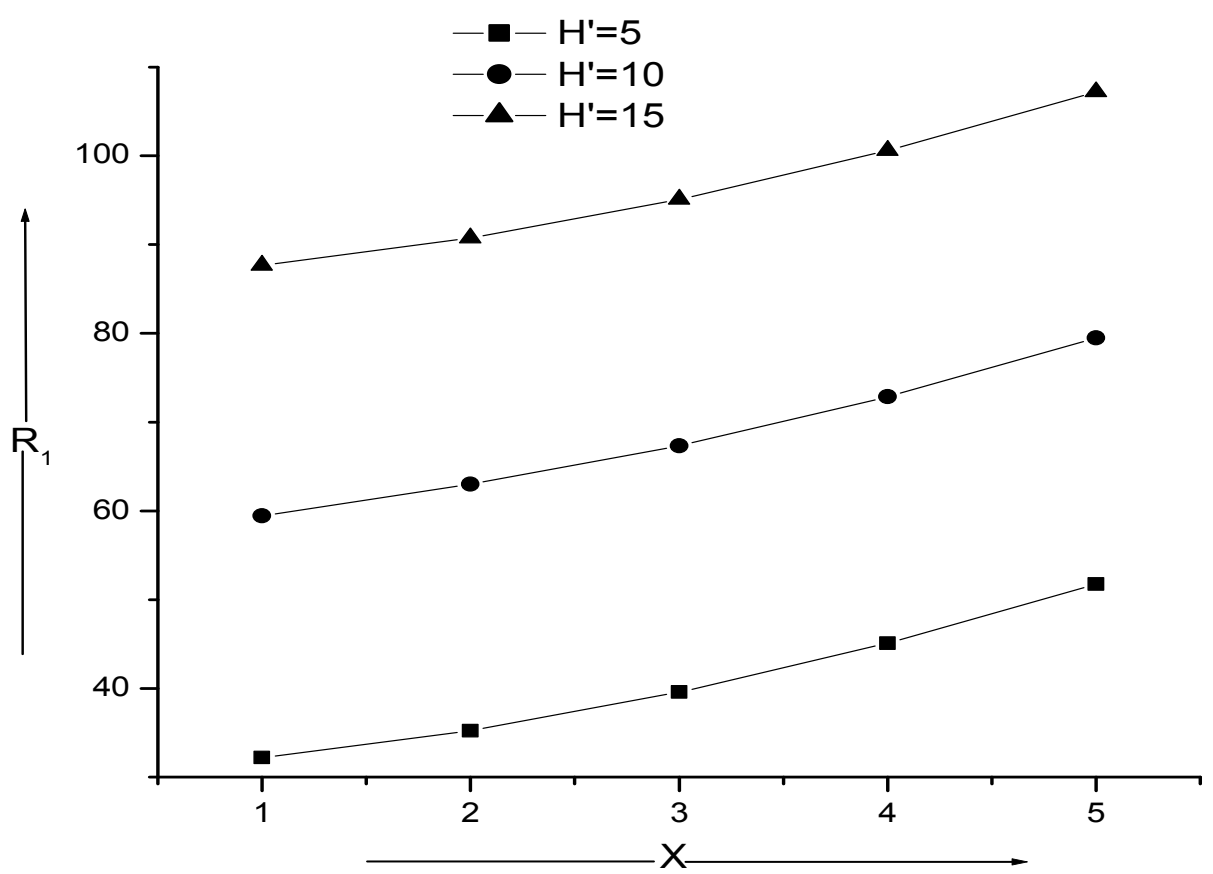

Fig.3. The variation of the Rayleigh number $\left(\mathrm{R}_{1}\right)$, with the wave number $x(=1,2,3,4,5)$, for $S_{1}=10$, $H=2, G=9.8$, and $H^{\prime}(=5,10,15)$. 
For fixed $S_{1}, H$ and $H^{\prime}$, let $G$ (accounting for the compressibility effects) also be kept fixed. Then we find that

$$
\bar{R}_{c}=\left(\frac{G}{G-1}\right) R_{c}
$$

where $\bar{R}_{c}$ and $R_{c}$ denote respectively the critical Rayleigh number in the presence and absence of compressibility. $G>1$ is relevant here. The case $G<1$ and $G=1$ correspond to negative and infinite values of the critical Rayleigh number in the presence of compressibility, which are not relevant in the present study. Compressibility thus postpones the onset of thermosolutal convection.

\section{Conclusion}

Thermosolutal convection in the presence of a compressible fluid with fine particles is considered. For stationary convection, the Rivlin-Ericksen, elastico-viscous fluid behaves like a Newtonian fluid. Suspended particles destabilize the system whereas the stable solute gradient has a stabilizing effect on the system and compressibility postpones the onset of thermosolutal convection. The stable solute gradient introduces oscillatory modes in the systems which were non-existent in its absence

\section{Nomenclature}

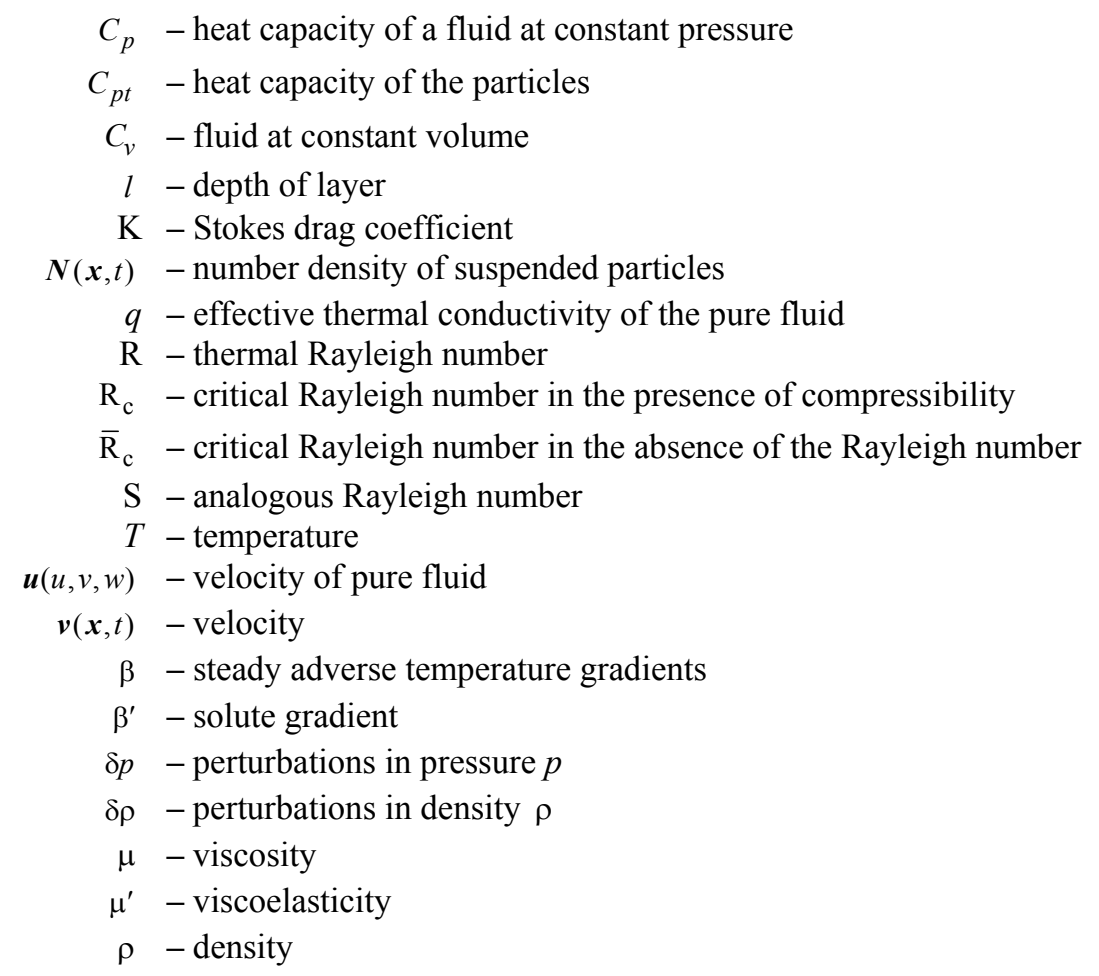

\section{References}

Chandra K. (1938): Instability of fluids heated from below. - Proc. Roy. Soc., London, vol.164A, pp.231.

Chandrasekhar S. (1981): Hydrodynamic and Hydromagnetic Stability. - New York: Dover Publication. 
Kumar P. (2000): Stability of superposed viscous-viscoelastic (Rivlin-Ericksen) fluids in the presence of suspended particles through a porous medium. - Z. Angew. Math. Phys., vol.51, pp.912-921.

Kumar P., Lal R. and Singh M. (2007): Hydrodynamic and hydromagnetic stability of two stratified Rivlin-Ericksen elastico-viscous superposed fluids. - Int. J. of Applied Mechanics and Engineering, vol.12, No.3, pp.645-653.

Kumar P., HariMohan and Lal R. (2006): Effect of magnetic field on thermal instability of a rotating Rivlin-Ericksen visco-elastic fluid. - International Journal of Mathematics and Mathematical Sciences (USA), ID 28042, pp.1-10.

Kumar P. and Singh G.J. (2010): On the stability of superposed viscous-viscoelastic fluids through porous medium. AAM (USA), vol.5(1), pp.110-119.

Palaniswami V.I. and Purushotham C.M. (1981): Stability of shear flow of stratified fluids with fine dust. - Physics Fluids, vol.24, pp.1224-1228.

Scanlon J.W. and Segel L.A. (1973): Some effects of suspended particles on the onset of Benard convection. - Physics Fluids, vol.16, pp.1573-1578.

Sharma R.C. and Rani N. (1989): Double-diffusive convection with fine dust. - Czechoslovak J. of Physics, vol.B39, pp.710.

Sharma V., Sunil and Gupta U. (2006): Stability of stratified elastico-viscous Walter's (B') fluid in the presence of horizontal magnetic field and rotation in porous medium. - Arch. Mech. Warszawa, vol.58, No.1, pp.187-197

Sharma R.C., Kumar P. and Sharma S. (2001): Rayleigh-Taylor instability of Rivlin-Ericksen elastico-viscous fluid through porous medium. - Indian J. Phys, 75B (4), pp.337-340.

Singh M. and Gupta R. (2011): Thermal instability of Rivlin-Ericksen elastico-viscous fluid permeated with suspended particles in hydrodynamics in a porous medium. - Int. J. of Applied Mechanics and Engineering, vol.16, No.4, pp.1169-1179.

Spiegel E.A. and Veronis G. (1960): On the Boussinesq approximation for a compressible fluid. - Astrophys J., vol.131, pp.442.

Received: June 13, 2012

Revised: October 29, 2013 